\title{
The Obstacle Avoidance for UAV Based on Improved Frames Difference and Optical Flow
}

\author{
Zhao Jin*, Bin Yan ${ }^{1}$ and Run Ye \\ ${ }^{1}$ University of Electronic Science and Technology of China, Chengdu, China \\ ${ }^{*}$ Corresponding author
}

\begin{abstract}
As to the outdoor real-time change has a bad influence on the obstacle detection and avoidance for UAV with monocular-version, this paper propose a moving obstacle detection algorithm based on three frames subtraction with adaptive iterative threshold and optical flow. Based on improved three frames subtraction, it can quickly separate the moving object from the UAV's forward version and simplify the complexity of the optical flow algorithm. By using optical flow method in the zone where moving obstacles detected, which effectively decreases the influence in brightness constant and calculates the closer obstacle to avoidance. Through the experiment, results show that this method can detect the moving object and calculate the obstacle need to avoidance in good real-time performance and robustness.
\end{abstract}

Keywords-lucas-kanade method; obstacle avoidance; frame difference; UAV

\section{INTRODUCTION}

With the technique in UAV (Unmanned aerial vehicle) highly developed, the UAV in civil application has been a focus in different fields. Therefore, the autonomous navigation by UAV generally be a hot topic to explore. By carrying varied sensors, UAV can adopt many different external environment and works [1].

Because of the unpredictable external environment, the autonomous navigation may be influenced by weather, lights and noise. Therefore, a stable and fast obstacle detection system is necessary. In another word, the monocular version with low cost and light weight can effectively avoid blindness of UAV. Therefore, the UAV with monocular camera gradually become a topic in obstacle avoidance research. But the monocular vision deeply relies on the contour of object and the environment. The influence may cause the unstable of autonomous, and cause the damage of UAV.

Considering the safety of the autonomous navigation and most of obstacle detection methods are complicating $[2,3]$. This paper proposes a potential obstacles detection system to eliminate the influence of lights and the low speed of the traditional detection algorithm. The main purpose of this paper is to simplify the region of UAV's fore-vision and reduce the influence of noise by using the frames difference with adaptive iterative threshold. And then, calculating the depth information of this regions with LK method to reduce the calculated quantities of traditional LK method. From the depth information of optical flow, the relative location and distance between UAV and obstacles can be estimated to avoid the obstacles. By using the adaptive iterative threshold, the frames difference can provide the accuracy object detection in time, and can fit for any environment. In the end, the experiment proves that the method combined LK and improved frames difference can be satisfied with any situation UAV worked for in time, and can accuracy detect the potential obstacles. Hence, the compound method is better than each single algorithm itself.

The structure of this paper is shown as follows. In section II, the research on recently monocular avoidance and the object detection. Section III and section IV are the details of the improved object detection. Section V and VI show the way of obstacle recognized and avoidance. From section VIII, the result will verify effectiveness of the algorithm. In the end section IX draws the conclusions.

\section{RELATED WORKS}

The key point for monocular version avoidance is to detect the moving obstacle in front of the UAV. And the moving object detection mainly contain background modeling and the depth information of optical flow [4]. Most the object detection algorithms paid more attention on the accuracy calculation of methods and the workspace indoor, instead of outdoors pace $[5,6]$. The modeling of background can effectively suit in simple background, and highlight the motion objects. Therefore, the combine of optical flow and modeling of background can help each other to overcome their disadvantage.

The criteria of frames difference is the accuracy of modeling for the foreground. The background modeling main contains mean, median and GAUSSIAN background modeling $[7,8,9]$. The main process of frames difference is to subtract every current frame with its former frame to extract the foreground from background $[10,11]$. But the effect always be affected by the modeling of changing background. The P-Tile is a semi- automatic adaptive threshold based on accumulation of gradation histogram, but need the percentage of object in background as the first condition [12]. The optimal threshold on trough has a great adaptive in frames with bimodal histogram, and through searching the valley in doublet to find the best threshold. But it doesn't fit for singlet frames or smooth frames [13]. Based on the Shannon entropy method, the adaptive threshold with the fuzzy set has an ideal result in the separation of frames in both doublet and singlet condition, but has no advantage in real time calculation [14].

The optical flow is a common way for objects detection by the optical vectors [15]. However, the optical flow calculation 
is quite huge. So, Lucas-Kanade optical flow was been put forward to calculate the sparse optical flow in sequential frame in 1981 [16]. Through doing research on the change of the frame gray, the method can analysis the corner motion and the depth information of the optical without the parameter of the camera, and can also avoid the update of the background and the time chosen among frames [17]. But large scale motion and the change of lights may cause the calculation with error in long range. Although the pyramid model can follow the iteration of different scaled frame to lessen the large-scale motion, the elements of lights still be the main problem of LK algorithm. Besides, the workspace of UAV usually spread in external environment. Therefore, the solution of lights and simplify computation can totally improve the online computing of the algorithm [18]. Learning from the insects, paper [19] simulate the insect's vision to detect the obstacle and avoidances in outside with low change of lights. Based on the ability of avoidance, the depth information of TTC (Time to Contact) and FOF (Focus of Expansion) accompanies with improved balance strategy are mentioned in an indoor space [20].

\section{IMPROVED FRAMES DIFFERENCE METHOD}

\section{A. Three Frames Difference}

From the idea of visual lag in human eyes, frame subtraction is to extract the delay from the vision to get the moving object. Because of the gray of the target is constant, it may cause big difference when the object is moving, whatever the speed it is. By comparing the difference and "and" operation of two subtractions in three adjacent frames, three frames difference can effective adopt the changing background and find the changing pixel to detect the moving object.

The details of the three frames difference can be described as: When get the vision frames, the method first get the gray pictures of 0,1 and so on. Then, the vision can be expressed as $\left\{F_{0}, F_{1}, \ldots, F_{k}, \ldots\right\}, F_{k}(x, y)$ is the $k_{t h}$ frames of the vision. What's more, the moving regions can be describe as:

$$
\begin{gathered}
D_{(k, k-1)}=\left|F_{k}(x, y)-F_{k-1}(x, y)\right| \\
D_{(k+1, k)}=\left|F_{k+1}(x, y)-F_{k}(x, y)\right|
\end{gathered}
$$

Where $D_{(k, k-1)}$ is the subtraction of $F_{k}(x, y)$ and $F_{k-1}(x, y)$.

Through threshold segmentation, the method can get the binary image of the foreground. After calculating two binary images of three consecutive frames, using "AND" operation to calculate as:

$$
T_{k}(x, y)= \begin{cases}1 & D_{(k, k-1)} \geq T \cap D_{(k+1, k)} \geq T \\ 0 & D<T\end{cases}
$$

Among formula (3), the $k_{t h}$ binary image shown as $T_{k}(x, y)$, and threshold be set as $T$. By setting an appropriate threshold, the influence of illumination can be ignored, and get an elaborate object.
For the problem of foreground's cavity. The paper adds the morphological method in the binarized difference images to lessen the calculation. By the way, removing noise in a convenient and fast way.

\section{B. The Adaptive Iterative Threshold}

As the unpredictable influence in external space, the threshold set by experience can't fit for the real-time environment. The setting standard for the threshold is to separate the object from the background based on the gray difference between them. If the gray lower than the threshold, it will be defined as a part of the object instead of background. The principle of the adaptive iterative threshold is by setting up an initial threshold to split the frame into background and foreground, then, calculating the threshold of each space, and so on. The terminal condition of this method is that the mean of background's gray and foreground's gray equals with the initial threshold.

With the changing of gradation histogram, the paper takes advantage of the order of gradation histogram, and then, calculates the frames with the iterative optimal threshold.

First, the method gets the gray level with maximum and minimum, and defines maximum as $g_{M}$, minimum as $g_{m}$. So, the threshold in initial iterative can be defined as:

$$
T_{\text {initial }}=\frac{g_{m}+g_{M}}{2}
$$

Then, through comparing $T_{\text {initial }}$ with the frame, the region which beyond the $T_{\text {initial }}$ is fore and the region which lower than $T_{\text {initial }}$ as back. And the mean of fore and back is shown as:

$$
M_{b}=\frac{\sum_{g=g_{m}}^{T_{\text {initial }}} g \times h(g)}{\sum_{g=g_{m}}^{T_{\text {intial }}} h(g)} \quad M_{f}=\frac{\sum_{g=T_{\text {intital }}}^{g_{M}} g \times h(g)}{\sum_{g=T_{\text {initial }}}^{g_{M}} h(g)}
$$

Where $g$ is the location of the gray level $h(g)$.

The $k_{t h}$ iterative threshold $T_{K}$ is:

$$
T_{K}=\frac{M_{f}+M_{b}}{2}
$$

Finally, judging the magnitude of $T_{K}$ and $T_{\text {initial. }}$. If the subtraction of $T_{K}$ and $T_{K-1}$ lower than the criteria, the iterative comes to the end, and $T_{K}$ is the optimal threshold. Or else, repeating the circulation until 100 times iteration. And the final threshold used in three frames differences can be defined as:

$$
\text { Threshold }=\frac{T_{\text {optimal }}}{C}
$$


$T_{\text {optimal }}$ is the finally results based on the adaptive threshold, and $C$ is a constant. Although the bigger threshold will reduce more noise in the frames, it will also cut down more details of the motion objects. Through plenty of experiments, the results prove that the best range of $C$ is among $[13,15]$. And the results not only effective cut down the noise, but also keep the contour of the motion objects clearly.

\section{LUCAS-KANADE METHOD}

Following the previous chapter, the frame difference method gets the area of moving objects. The next step is to calculate the optical flow changing of this area. The Lucas-Kanade method is a way good at computing sparse optical flow in a local field information.

First, the method should follow the limitation 'brightness constancy', 'time-continuous' and 'space consistency' [21]. Then, assume that the brightness point can be described as $I(x, y)$. According to 'brightness consistency', the point is constant over time:

$$
I(x, y, t)=I(x+\delta x, y+\delta y, t+\delta t)
$$

Where $\delta x, \delta y$ stand for the shifts between continuous frames among $\delta t$ period. By Taylor series expansion, formula (9) can be expressed as:

$$
\frac{\partial I}{\partial x} \frac{d x}{d t}+\frac{\partial I}{\partial y} \frac{d y}{d t}+\frac{\partial I}{\partial t}=0
$$

Where

$$
\frac{d x}{d t}=u \quad \frac{d y}{d t}=v \quad \frac{\partial I}{\partial x}=I_{x}, \frac{\partial I}{\partial y}=I_{y}
$$

And the optical flow vector $\bar{v}$ can be $(u, v)$.

Considering about 'space consistency', the velocity vector stay slight in successive pictures, Formula (1) can be Taylor expand as:

$$
I(x, y, t)=I(x, y, t)+(\nabla I)^{T}(\delta x, \delta y)+\delta t I_{t}+O^{2}
$$

Where, $\nabla I=\left(I_{x}, I_{y}\right)$ and $I_{t}$ is the derivative of time $t$, $\mathrm{O}^{2}$ represents for the infinitesimal of high order. Though subtracting both side of equation (12) and simplify, the result shows that:

$$
(\nabla I)^{T} \bar{v}+I_{t}=0
$$

Defining functions, $F(x)$ is the value about each frame's gray and $G(x)=F(x+h), h$ is the vector displacement. Therefore, the gradient of $F(x)$ can be written as:

$$
F^{\prime}(x)=\frac{F(x+h)-F(x)}{h}=\frac{G(x)-F(x)}{h}
$$

And the error estimates of optical flow can be shown as:

$$
E=\sum_{x}(F(x+h)-G(x))^{2}=0
$$

For the minimize error, calculating the extremum of $E$

$$
\frac{\partial E}{\partial h}=0
$$

After abbreviation, $h$ is written as:

$$
h=\frac{\sum_{x} F^{\prime}(x)[G(x)-F(x)]}{\sum_{x} F^{\prime}(x)^{2}}
$$

Since the shift of $h$ is low, combining the LK method with pyramid model. By setting up frames in different multiscale, iteration starts from minimum scaled frames (top of pyramid) to the origin frames (bottom of the pyramid). According to the pyramid LK method, the main disadvantage of LK (losing large scale motion) can be overcome. And the process of the Pyramid LK shown as Figure1.

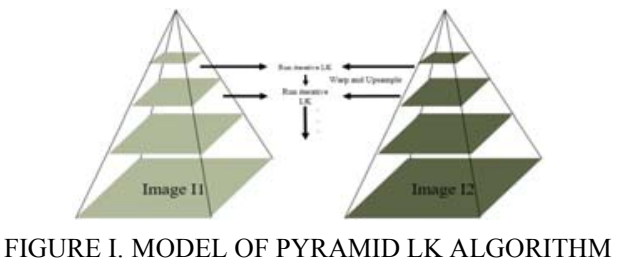

Usually, it's about 3 levels pyramid. $L_{1}=320 \times 240, L_{2}=$ $160 \times 120, L_{3}=80 \times 60$. At each frame, for the best optical flow $\bar{v}$, function (13), the gradient of $\nabla I$ as

$$
\nabla I \dot{=}\left[\frac{\partial F}{\partial x} \frac{\partial F}{\partial y}\right]^{T}
$$

What's more, formula (16) as follows:

$$
\sum_{x}\left(\nabla I^{T} \bar{v}-\delta I\right) \nabla I=0
$$

By substituting (15), it shows:

$$
\sum_{x}\left(\left|\begin{array}{ll}
F_{x}^{2} & F_{x} F_{y} \\
F_{x} F_{y} & F_{y}^{2}
\end{array}\right| \bar{v}-\left|\begin{array}{c}
\delta I F_{x} \\
\delta I F_{y}
\end{array}\right|\right)=0
$$




$$
G=\sum_{x}\left(\left|\begin{array}{lr}
F_{x}^{2} & F_{x} F_{y} \\
F_{x} F_{y} & F_{y}^{2}
\end{array}\right|\right), \quad b^{-}=\sum_{x}\left(\left|\begin{array}{l}
\delta I F_{x} \\
\delta I F_{y}
\end{array}\right|\right)
$$

Finally, the best optical flow vector has been gotten:

$$
\bar{v}=G^{-1} \bar{b}
$$

For the $G$ be nonsingular in LK tracking, the parper uses Shi-Tomasi to calculate the corner[22].

\section{V.The Motion Obstacle Detection}

As is known to all, the object closer to you will have a faster speed instead of the distant view. What's more, the closer object will also take up much more vision, and generate bigger optical flow vector. According to this principle of motion parallax, the paper uses the depth information of optical flow calculated last chapter and the direction of UAV attitude control to let UAV avoid the potential risks. First, the vision is separated into upward, downward, left side and right side. The field is shown as Figure2. By the way, getting the mean flow of each field.

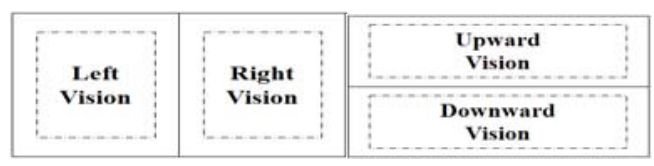

FIGURE II. THE SEPARATION OF OPTICAL FIELD

Whether the moving object is a potential risk or not, it is judged by the next formula:

$$
u_{i}=1-\frac{1}{\left\|O F_{i}\right\|} \frac{\sum\|w\|}{N}
$$

Where $\left\|O F_{i}\right\|$ is the modular of the $i^{\text {th }}$ corner, and $\sum\|w\|$ is the modular of the opposite area about the $i^{\text {th }}$ corner. $N$ stands for the number of the area. By setting threshold $T$, when the modular in $i^{\text {th }}$ area beyond double of $T$, it's been defined as area where has potential risks. Instead, $u_{i}>2 T$ shows that the area may have obstacles but can't be a threat.

\section{Description of the ObStacle Avoidance}

Being related to the separated vision, this employs the laws of optical flow balance [23]. The principle means that the UAV will take avoidance method by far away the region where has higher optical flow modular. By setting up four oriented, the UAV can control pitch and roll posture control to avoidance obstacle. The paper defines the mean value of each region optical flow as the judgement criteria. Take the horizontal avoidance as example:

$$
\Delta F=1-\frac{m}{n} \frac{\sum\left\|w_{\min }\right\|}{\sum\left\|w_{\max }\right\|}
$$

$\sum\left\|w_{\max }\right\|$ is the region with less modular and $m$ is the total number of this region's optical flow vectors, instead $\sum\left\|w_{\text {min }}\right\|$ and $n$. If $\Delta F>T$, it means that the region with higher optical flow has danger. Hence, taking Law of Control to transform the difference of optical flow to the UAV's control:

$$
\Delta\left(F_{L}-F_{R}\right)=k \frac{\sum\left\|w_{L}\right\|-\sum\left\|w_{R}\right\|}{\sum\left\|w_{L}\right\|+\sum\left\|w_{R}\right\|}
$$

Where $\Delta\left(F_{L}-F_{R}\right)$ means the difference of roll command, and $k$ is a transform constant. According to the function (25), the drone will get the accuracy command to change its attitude.

\section{The OBstacles AVOIDANCE BAESD ON LK METHOD AND THREE FRAMES DIFFERENCE}

For the accuracy and adaptive in real-time environment, this paper combined LK optical flow and the three frames difference with adaptive iterative threshold to detect the object in potential risks and avoid. The algorithm can be described as:

Step1. Setting the real frame and its two border frames, and then using the three frames with adaptive iterative threshold to detect the motion region and simplify the fore-vision.

Step2. According the motion object region to apply the LK method to calculate the optical flow vectors as function (21). And keep tracking the moving objects.

Step3. Through operation (22) to judge whether the fore-vision has the potential risks or not. If there are some potential obstacles, researching the optical flow fields it belongs to. If not, return to step1.

Step4. Executing the avoidance orders based on function (22), (23) until there is no obstacle in front of the UAV. Back to the step1.

\section{SIMULATION RESULTS}

In order to prove the validity of the combined method, the paper uses VS 2015 platform and OpenCV 3.0 as the ground station to test the algorithm, and then sends attitude orders through PWM signals to UAV to test avoidance in real time condition, the speed of UAV is $1.2 \mathrm{~m} / \mathrm{s}$, the common test environment as figure 3 shows.
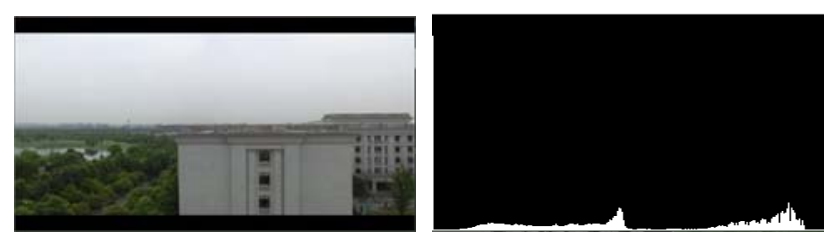

FIGURE III. REAL-TIME ENVIRONMENT AND ITS GRADATION HISTOGRAM

After using three frames difference with adaptive iterative threshold and morphology filter, the method can quickly adaptive the environment of UAV's fore-vision and capture 
the moving obstacles with its locations. What's more, the adaptive iterative threshold effectively reduces the noise from the background and natural factors and gets the contour of moving objects. The results in Figure 4 show that three frames difference effectively simplify the optical flow detection environment and keep fore-vision stable.

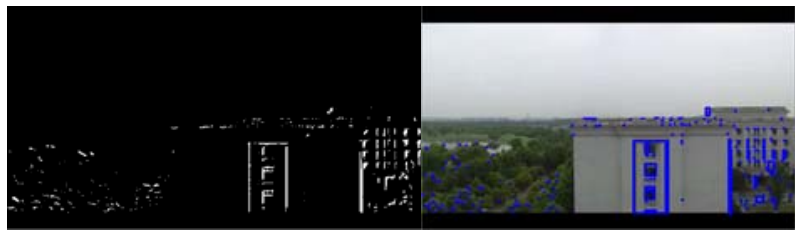

FIGURE IV. DETECTION OF THREE FRAMES DIFFERENCE

Because of the simplify of environment, compared Figure 3(a), the method combined LK and improved frames difference shown in 3(b) effectively reduces the noise, and provides the region for the pyramid LK method to track. It is obvious that the method improves the accuracy of potential obstacles detection and complexity of the optical flow algorithm.
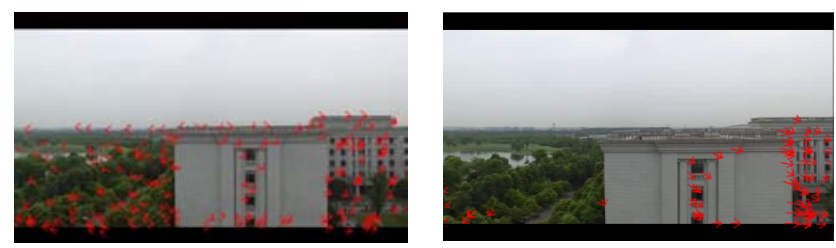

FIGURE V. (a) THE TRADITIONAL LK METHOD, (b) THE COMBINED LK METHOD

From Figure 5(b), the result shows that the right and downward version have quit higher optical flow in UAV's forevision. Through function(23), (24), the results show that the obstacles in the bottom right of UAV, but can not be a threat and the UAV can keep going.

Considering the environment with high fill factor, the frame as Figure 6 shown, the exposure cause the main influcence for UAV navigation, and the vision can't distinguish the object exactly.

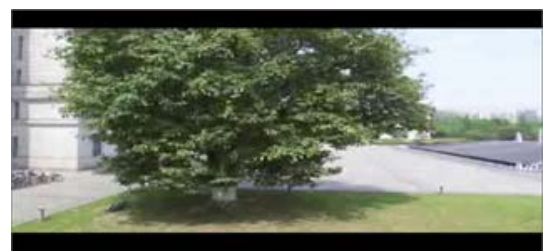

FIGURE VI. HIGH FILL ENVIRONMENT

With the speed of $2.4 \mathrm{~m} / \mathrm{s}$ and height is $3 \mathrm{~m}$ in hihg exposure region, the UAV's fore-vision with combined method is shown as Figure 5.

From the Figure 7, the results show that the three frames difference can effectively overcome the influence with illumination, and provides the accuracy region for the depth information of the potential obstacles. According to the optical flow vectors and function (23), (24), (25), the $u_{i}>2 T$, which means that UAV's left side has potential obstacles and should change the angle of roll to let UAV turn right.

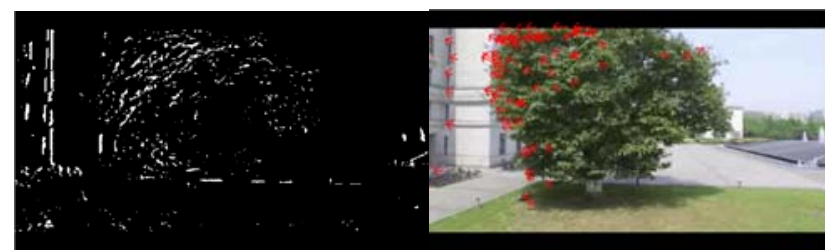

FIGURE VII. (a) THREE FRAMES DIFFERENCE, (b) PYRAMID LK METHOD

\section{CONCLUSIONS}

This paper main to eliminate the illumination and noise influence when UAV is taking the autonomous navigation. First, the paper uses the three frames difference with adaptive iterative threshold to simplify the environment to detect moving objects and uses morphology to erase the influence of lights. Then, the pyramid LK method will track the region detected by frames difference and calculate the optical flow in each region to control UAV avoidance online. The results show that the frames difference algorithm can effectively reduce the influence of lights and shrink the object regions. And the calculation of pyramid LK method in simplify region has improved the speed to detect the obstacles. What's more, it can adopt the environment quickly and ensure the safety of autonomous navigation.

\section{ACKNOWLEDGEMENT}

During the time of my researching, I would give my all gratitude to the teachers and mates whoever helped me. And I'll also give my thanks to the professor Xiaojia Zhou, Bin Yan. Last, I will give my thanks to doctor Ye for the suggestion and guidance in my research.

\section{REFERENCES}

[1]. Ugur Cekme1, Mustafa Ozsiginan and Ozgur Koray Sahingoz, "A UAV PATH PLANNING WITH PARALLEL ACO ALGORITHM ON CUDA PLATFORM," 2014 International Conference on Unmanned Aircraft Systems (ICUAS), 2014, pp. 347-354

[2]. I. Ulrich and I.R. Nourbakhsh, "Appearance-based obstacle detection with monocular color vision," in AAAI/IAAI, 2000

[3]. Souhila, K. and Karim, A. "Optical Flow Based Robot Obstacle Avoidance," International Journal of Advanced Robotic Systems, 2007, pp. 13-16.

[4]. Xin-Zhong Peng, Huei-Yung Lin, and Jyun-Min Dai, "Path Planning and Obstacle Avoidance for Vision Guided Quadrotor UAV Navigation," 12th IEEE International Conference on Control \& Automation (ICCA), 2016, pp. 984-989.

[5]. Milton C. P. Santos, Lucas V. Santana, Alexandre S. Brandao and Mario Sarcinelli-Filho, "UAV Obstacle Avoidance using RGB-D System," 2015 Internal Conference on Unmanned Aircraft Systems (ICUAS), 2015, pp. 312-319

[6]. A. Giachetti, M. Camani, V. Torre, "The Use of Optical Flow for Road Navigation," IEEE Transactions on Robotics and Automation, vol.14, Issue 1, 1998, pp.34-48

[7]. Zdzisław Gosiewskil, Jakub Cieśluk1, Leszek Ambroziak1, "Vision-based obstacle avoidance for Unmanned Aerial Vehicles," 2011 4th International Congress on Image and Signal Processing, 2011, pp. 2020-2025

[8]. Chalidabhongse T H. Kim K. Harwood D. et al, "A perturbation method for evaluating background subtraction algorithms", Proceeding of the joint IEEE international workshop on visual surveillance and performance evaluation of tracking and surveillance, 2003, pp. $11-12$.

[9]. Stauffer C, Grimson W, "Adaptive background mixture models for real - time tracking," Proceeding of 1999 IEEE international conference on 
computer vision and pattern recognition. 1999, pp. 246 - 252 .

[10]. Tsai Y M, Tsai C C, Huang K Y. et al, "An intelligent vision based vehicle detection and tracking system for automotive applications," Proceedings of 2011 IEEE international conference on consumer electronics, 2011, pp. $113-114$

[11]. Feng Yuxi, He Guotian, Wu Qizhou. "A New Motion Obstacle Detection based Monocular-Vision Algorithm," 2016 International Conference on Computational Intelligence and Applications, 2016, pp. 31-35

[12]. Fan, Jiu Lun, W. Z. Zhen, and W. X. Xie. "Suppressed fuzzy c -means clustering algorithm.” Pattern Recognition Letters. Vol. 24, 2003, pp. 1607-1612.

[13]. C. A. Glasbey, "An analysis of histogram-based thresholding algorithms," CVGIP: Graphical Models and Image Processing, vol. 55, 1993, pp. 532-537

[14]. liang-kai Huang, Mao-jiun J. Wang, "Image Threshodling By Minizing The Measure Of Fuzziness, "Pattern Recognition vol. 28, 1995, pp. 41-51

[15]. Xiaowei Han, Yuan Gao, Zheng Lu, Zhimin Zhang, Dun Niu, "Research on moving object detection algorithm based on improved three frame difference method and optical flow," 2015 Fifth International Conference on Instrumentation and Measurement, Computer, Communication and Control, 2015, pp. 580-584

[16]. Angel Juan Sanchez-Garcia, Homero Vladimir Rios-Figueroa .et al, "Decision Making for Obstacle Avoidance in Autonomous Mobile Robots by Time to Contact and Optical Flow," 2015 IEEE, 2015, pp. 130-134

[17]. H. Kim, C. Ahn, G. Jeong, M. Kim and K. Sun, "Vessel Boundary Detection for its 3D Reconstruction by Using a Deformable Modeling (GVF Snake)," International Conference of the Engineering in Medicine \& Biology Society, 2015, pp. 3440-3445.

[18]. B. D. Lucas and T. Kanade. "An iterative image- registration technique with an application to stereo vision," Proceedings of the 1981 DARPA Imaging Understanding Workshop, 1981, pp. 121-130

[19]. Hongyan Huang, Siwen Gao, Yanmei Yu, Xiaohai He, "Visual Obstacle Avoidance System Based on Optical Flow Method," Journal of Image and Signal Processing, 2016, pp. 66-72

[20]. Chaoqun Wang, Wei Liu, Max Q. H. Meng, "Obstacle Avoidance for Quadrotor Using Improved Method Based on Optical flow," International Conference on Information and Automation, 2015, pp.1674-1679

[21]. VIET VO, NGOC LY, “An effective approach for human actions recognition based on optical flow and edge features," ICCAIS2012, Ho Chi Minh City, 2012, pp. 24-29

[22]. Shi, J. and Tomasi, C. "Good Features to Track", Proceedings of the IEEE Conference on Computer Vision and Pattern Recognition, Seattle, 1994, pp. 593-600.

[23]. Fraid Kendoul, Isabelle Fantoni, Kenzo Nonami, “Optical flow-based vision system for autonomous $3 \mathrm{D}$ localization and control of small aerial vehicles”, Robotics and Autonomous System, 2009, pp. 591-602 